\title{
Fixed Pattern Noise Analysis for Feature Descriptors in CMOS APS Images
}

\author{
Juan Zapata-Pérez ${ }^{\mathrm{a}}$, Ginés Doménech-Asensi ${ }^{\mathrm{a}}$, Ramón Ruiz-Merino ${ }^{\mathrm{a}}$, Jose \\ Javier Martínez-Álvarez ${ }^{\mathrm{a}}$, Ricardo Carmona-Galán ${ }^{\mathrm{b}}$, Jorge \\ Fernández-Berni ${ }^{\mathrm{b}}$ \\ ${ }^{a}$ Dpto. de Electrónica, Tecnología de Computadoras y Proyectos \\ Univ. Politécnica Cartagena (Spain) \\ ${ }^{b}$ Instituto de Microelectrónica de Sevilla, IMSE-CNM, CSIC-Universidad de Sevilla
}

(Spain)

\begin{abstract}
This paper provides a comparative performance evaluation of local features for images from CMOS APS sensors affected by fixed pattern noise for different combinations of common detectors and descriptors. Although numerous studies report comparisons of local features designed for ordinary visual images, their performance on images with fixed pattern noise is far less assessed. The goal of this work is to develop a tool that allows to evaluate the performance of computer vision algorithms and their implementations subject to deviations of the physical parameters of the CMOS sensor. This tool will facilitate the cuantification of the high-level effects produced by circuit random noise, enabling the optimization of the sensor during the design flow with specifications much closer to the application scope. Likewise, this tool will provide the electronic designer with a relationship between high-level algorithm accuracy and maximum fixed pattern noise. Thus the contribution is two-fold: i) to evaluate the performance of both local float type and more recent binary type detectors and descriptors when combined under a variety of image transformations, and ii) to extract relevant information from circuit-level simulation and to build a noise model to be employed in the design of the feature descriptor evaluation. The utility of this approach is illustrated by the evaluation of the effect of column-wise and pixel-wise fixed pattern noise at the sensor on the performance of different local feature descriptors.
\end{abstract}

\footnotetext{
*Corresponding author. Tel.: +34 9683264 58. E-mail: juan.zapata@upct.es
} 
Keywords:

CMOS Image Sensors, 3T-APS Pixels, Fixed Pattern Noise, Local Feature Descriptors, Homography Transformation

2

\section{Introduction}

The emergence of powerful, low-cost, and energy-efficient processors has enabled the incorporation of practical computer vision capabilities in embedded systems, mobile devices, PCs, and the cloud. As a result, in the coming years, there will be a rapid proliferation of integrated vision technologies in many different application scenarios. In this context, we can define a smart camera as an autonomous vision system integrating an image sensor and all the required electronics to analyze the targeted scene. Concerning the image sensor, while analog realizations could still be in use, we focus in this work on digital image sensors, typically from a Charge-Coupled Device (CCD) or a Complementary Metal-Oxide-Semiconductor (CMOS) sensor array that operates with visible light - though many vision systems can also detect other types of energy (IR, sonar, etc). CCD sensors exhibit some advantages over CMOS image sensors in terms of image quality and dynamic range. However, our interest lies in CMOS image sensors, which now account for more than $90 \%$ of the market ${ }^{1}$. This predominance is heavily influenced by the massive market of camera phones demanding lower cost, better integration and higher speed. In CMOS Active Pixel Sensor (APS) imagers [1], the performance of the pixel depends on technology parameters and the physical design rules of the manufacturing process. Thus, the enhancement of pixel performance can be achieved by optimizing process-related parameters. This in turn calls for the development of technology-based models to evaluate particular processes and to optimize the electrical and physical performance of the imaging pixel.

The physical implementation of different primitives for capturing and processing the image suffers from deviations from the ideal behavior. On the one hand, the implementation of a sensor is not free of noise sources. For example, the reset operation gives rise to thermal noise in a three-transistor active pixel sensor (3T APS) shown in Figure $1[2,3]$. The photodiode has to be reset before the charge accumulation begins again, so that in each new cycle the photodiode reset circuit forms a low pass filter $\mathrm{RC}$ with the equivalent

\footnotetext{
${ }^{1}$ https://www.embedded-vision.com/technology/cameras-sensors
} 
resistance of the reset transistor on, generating a component of noise kTC. Another example is found in the transistors that act as a voltage follower in the same 3T-APS pixel, which show across-chip deviations in their threshold voltage [3]. This introduces a permanent deviation that is specific to each pixel. It is known as fixed pattern noise (FPN). On the other hand, the accuracy of many computer vision algorithms, such as the extraction of local feature descriptors in images, can be sensitive to variations in the parameters of the operation. For this reason, in most cases, the function implemented is by itself a feasible approximation of the function ideally contemplated in the original design of the vision algorithm.

Taking into account that power consumption and algorithmic accuracy must be properly balanced in embedded devices, it is essential to carry out a detailed analysis of the relation between the CMOS image sensor performance and high-level computer vision tasks within the system design cycle. Overall, in this paper, we present a method which relates the performance of different computer vision feature extraction algorithms with FPN. The aim of this method is to provide electronic designers with a tool able to set the maximum value of FPN allowed to meet a given algorithm accuracy specification. Section 2 describes works related to noise modeling, 3T-APS technology and local features descriptors. In Section 3 the experimental methodology that has been used to perform the proposed analysis by means of image processing techniques is detailed. The results obtained for both binary and floating-point descriptors are discussed in Section 4. Finally we conclude in Section 5.

\section{Related work}

An APS is an image sensor where each picture element or pixel has a photo-detector and an active amplifier. The term active pixel sensor was coined in 1985 by Tsutomu Nakamura et al. [4] and more broadly defined by Eric Fossum [5]. The term 'active pixel sensor' is also used to refer to the individual pixel sensor itself, as opposed to the image sensor [6]; in that case the image sensor is sometimes called an active pixel sensor imager [7], or active-pixel image sensor [8]. There are many types of active pixel sensors, including CMOS APS used most commonly in cell phone cameras, web cameras, most digital pocket cameras since 2010, and in most digital single-lens reflex cameras (DSLRs). Such an image sensor is produced using CMOS 
technology (and is hence also known as a CMOS sensor), and has emerged as an alternative to CCD image sensors.

The APS sensor amplifies the signal in each pixel by converting the charge accumulated into a gate potential. For the 3T-APS configuration in Figure 1, the transistor $M_{s f}$ acts as a voltage follower, so that the output voltage follows the voltage of the photo-diode. The signal is transmitted to the vertical output line through the selection transistor $M_{s e l}$. The operation of this sensor is as follows [9]: i) The reset transistor $M_{r s t}$ is set on by means of the horizontal input line RST. The photo-diode is reset to $V_{D D}-V_{t h}$, where $V_{t h}$ is the threshold voltage of $M_{r s t}$; ii) $M_{r s t}$ is set off; iii) under an incident light source, the photo-diode accumulates photo-generated carriers in the sensing capacitor $C_{i n t}$. These carriers modify the potential in the photo-diode which decreases with respect to the intensity of the incident light, which decreases as a function of the incident light; iv) after a given $t_{e x p}$ exposure time, the selection transistor $M_{\text {sel }}$ is activated by means of the horizontal input line ROW and the output voltage of the pixel is read on the vertical output line COL; v) once the reading process is finished, $M_{s e l}$ is deactivated and $M_{r s t}$ is activated, returning to point i).

[Figure 1 about here.]

Previous works $[10,11]$ have tried to evaluate the performance of hardware for software codesign modeling some physical errors. In the case of image sensors, those physical errors are mainly manifested as noise. The European Machine Vision Association (EMVA) standard 1288 [12] defines the EMVA 1288 Standard [13] in order to measure, compute and present specification parameters and characterization data for cameras and image sensors used for machine vision applications. The general assumptions covered by the standard include: i) the amount of photons collected by a pixel depends on the product of irradiance $E$ (units $\mathrm{W} / \mathrm{m}^{2}$ ) and exposure time $t_{\exp }$ (units $\mathrm{s}$ ), i.e., the radiative energy density $E t_{\text {exp }}$ at the sensor plane; ii) the sensor is linear, i.e., the digital signal $y$ increases linearly with the number of photons received; iii) all noise sources are wide-sense stationary and white with respect to time and space, i.e., the parameters describing the noise are invariant with respect to time and space; iv) only the total quantum efficiency is wavelength dependent, i.e, the effects caused by light of different wavelengths can be linearly superimposed; and v) only the dark current is temperature dependent. 
These assumptions describe the properties of an ideal camera or sensor, as shown in Figure 2. A real sensor will depart more or less from an ideal sensor. As long as the deviation is small, the description is still valid, and it is one of the tasks of the standard to describe the degree of deviation from an ideal behaviour. In this work, it is assumed that the image pixel sensor to be simulated complies with the necessary features to be described by the mathematical pixel model proposed by the standard. This mathematical model tries to follow the physical model of an image sensor. The idea is that a digital image sensor essentially converts photons hitting the pixel area during the exposure time by a sequence of steps finally into a digital number. That sequence is as follows: a number of photons $\left(\mu_{p}\right)$ hitting the pixel area during exposure time $\left(t_{\text {exp }}\right)$ creates a number of electrons $\left(\mu_{e}\right)$ determined by the quantum efficiency $(\eta)$. These electrons, together with those that are product of noise and other device non-idealities $\left(\mu_{d}\right)$ form a charge which is converted by a capacitor to a voltage. This voltage is then amplified with a gain $(K)$ and digitized by means an analog-digital converter (ADC) that introduces a quantization noise $\left(\sigma_{q}\right)$. At the end of this process the result is a digital grey value $(y)$.

At the highest level of abstraction for this grey digital image, a keypoint is a small portion of the image that, for any reason, is unusually distinctive, and can be easily identified in a related image. A descriptor is some mathematical construction, typically a vector of floating-point or binary values, which somehow describes an individual keypoint, and which can be used to determine whether -in some context- two keypoints from two different images correspond to a same feature. There are roughly two categories of descriptors. On the one hand, there are floating-point descriptors based on the Histogram of Gradients (HOG), that is, SIFT [14], SURF [15], VGG [16] and Daisy [17]. On the other hand, there are the binary descriptors, BRISK [18], BRIEF [19], ORB [20], LATCH [21], FREAK [22], KAZE [23], AKAZE [24], among others that have demonstrated to be successful alternatives that provide similar performance to their floating point counterparts while being efficient to calculate and store. In this paper, a comprehensive evaluation of the performance of cutting-edge binary descriptors, that is, BRIEF (ORB), BRISK, and those based on Histogram of Gradients such as SIFT and SURF, is presented in the context of image matching under various geometric and photometric image transformations.

[Figure 2 about here.] 


\section{Experimental methodology}

Our aim is to match two complex images, containing heterogeneous objects that are arranged in the background, by means of a matrix of feature descriptors. Each descriptor is a vector which is linked to a keypoint, and each element of the vector can be a binary or a non-binary value. The image is contaminated by a model of fixed pattern noise based on EMVA standard 1288. We want to assess how this fixed pattern noise hinders the matching operation. A keypoint will be considered a true positive if the keypoint is an inlier of a homography transformation between images. The outliers will be considered false positives. The noise contribution of main interest for us is the column-wise fixed pattern noise since it is created in CMOS imagers which include column-parallel ADC readout architectures.

The process of matching two images consists in comparing every feature in the image A to every feature in the image B, in order to detect which keypoints refer to the same feature in both images. Three strategies can be used if the distance is the metric used to assess the matching: brute force using near neighbour distance rate (NNDR), cross checking and k-d trees or hash tables. To this end, once some hypothetical or predictive matches have been obtained, geometric alignment (homography tranform) is used to verify which matches are inliers (true positives) and which ones are outliers (false positives). The followed procedure involves four stages, as shown in Figure 3. Stage 1 consists of a noise model which is added to the image. Stage 2 performs the extraction and description of the features of the images. Stage 3 carries out a matching stage, where the images are compared using the three aforementioned matching strategies. Stage 4 uses a homography transformation to obtain a fit of only those features matches (inliers) that are sufficiently close to this estimated transformation.

[Figure 3 about here.]

\subsection{Modeling fixed pattern noise}

The noise contribution of main interest for us is the fixed pattern noise (FPN) which is the variation in output pixel values, under uniform illumination, due to device and interconnect mismatches across an image sensor. It is fixed for a given sensor, but varies from sensor to sensor. FPN consists of offset and gain components. It increases with illumination, but causes more degradation in image quality at low illumination. In a CCD image 
sensor, FPN is modeled as a sample from a spatial white noise process. A spatial white noise random process is a set of zero-mean uncorrelated random variables with the same standard deviation. If the random variables are Gaussian, the noise is completely specified by standard deviation. This white noise model is not suitable for characterizing FPN in CMOS sensors due to the strong differences of the readout circuitry used in CMOS sensors and CCDs. Both CMOS passive pixel sensor (PPS) and APS have higher FPN than CCDs and suffer from column FPN, which appears as "stripes" in the image and can result in significant image quality degradation. In CMOS image sensors, pixel transistors cause additional pixel FPN while column amplifiers cause column FPN. As a result, FPN is in general higher in CMOS than in CCDs. Therefore, we represent FPN as the sum of two components: column-level and pixel-level.

Pixel-wise and column-wise FPN are mainly caused by the variation in the photodetector parameters and dark current. In APS it is mainly caused by variations in transistor parameters. Both FPN noise terms, are related with the operation of the source follower, $M_{s f}$, which in a first approximation provides an output voltage given by:

$$
V_{\text {out }}=V_{\text {pix }}-V_{T_{s f}}-\sqrt{I_{B} / \beta_{s f}}
$$

where $V_{p i x}$ is the pixel voltage, $I_{B}$ is the column bus current and $\beta_{S F}$ is the saturation transconductance factor for $M_{S F}$. This is the FPN term that occurs in a single pixel independently from the rest of pixels. It was shown in a seminal paper by Pelgrom et al. in 1989 [25] that, in any given MOS technology, the variance of the transistor threshold voltage $V_{T}$ (threshold mismatch) among adjacent transistors reduces inversely with the gate area. This is known as Pelgrom's law:

$$
\sigma_{V_{T}}^{2}=A_{V_{T}}^{2} / 2 W_{s f} L_{s f}
$$

where $A_{V_{T}}$ is a constant named Pelgrom's model constant that depends only on the process, $W_{s f}$ is SF transistor width and $L_{s f}$ is the $M_{s f}$ transistor length. Conversion gain $(K)$ expresses, in volts per unit charge, how much voltage change is obtained by one electron at the charge detection node. Thus, in terms of noise contributions, it can be incorporated to the model as:

$$
\sigma_{\Delta V_{T}}^{2}=\left(1 / K^{2}\right) A_{V_{T}}^{2} / 2 W_{s f} L_{s f}
$$


For column-wise FPN, variations of the column bus current $\left(I_{B}\right)$ from $M_{\text {sel }}$ introduce an additional offset. Its contribution in electrons is:

$$
\sigma_{\Delta I_{Q}}^{2}=\frac{I_{B}}{4 \beta_{s f} K^{2}}\left(\frac{A_{\beta}^{2}}{W_{B} L_{B}}+4 \frac{\beta_{B}}{I_{B}} \frac{A_{V_{T}}^{2}}{W_{B} L_{B}}\right)
$$

where $\beta_{B}$ is the saturation transconductance factor for bias, $W_{B}$ and $L_{B}$ is the width and length from transistor bias $M_{\text {sel }}$, and $A_{\beta}^{2}$ is the Polgrom's model constant.

\subsection{Feature descriptors}

A corner is a small patch of an image that is rich in local information. A keypoint is a small patch of an image that is rich in local information which is unique. Local information is stored in the form of a descriptor, which exhibits lower dimensionality than keypoint features. The idea behind this is that descriptor is much easier to recognize, unique o nearly unique, when it appears in another different image. The earliest ancestor of the modern keypoint is the Harris corner [26] which is the predecessor of the modern keypoints as well as their descriptors. The idea of Harris corner is that there are many kinds of local features (edges, grey levels, gradients, etc) that can be detected although in practice, the point or feature to be selected should be: 1) unique, or nearly unique in order to have a good chance of finding that point again and; 2) parameterizable in order to be compared.

Harris corners are rotation-invariant, which means that even if the image is rotated, the same corners can be found. Another important property is scale invariability. But a corner may not be seen as a corner if the image is scaled, due to possible losses of some strong derivatives in the scaled patch. In 2004, David Lowe [14] proposed a new algorithm to obtain scale invariant keypoints: the Scale-Invariant Feature Transform (SIFT). Lowe introduced a new property to the SIFT features to assure that the features would be distinctive. The SIFT algorithm extracts keypoints and compute their descriptors. The procedure can be divided in two main computational operators: keypoints detector and keypoints descriptor. The SURF algorithm (SpeededUp Robust Features) was originally proposed in 2006 by Bay et al. [15, 27]. SURF is also based on taking histograms of gradients orientation in the same way as SIFT. The algorithm can be understood as an evolution of the SIFT feature discussed just above. 
SIFT and SURF features are notable examples of the family of Histograms of Oriented Gradients (HOG) based descriptors. Although highly discriminative and robust to various image transformations, HoG based features rely on computationally expensive gradient histogram pooling and results in a high dimensional feature vector of floating-point values. To decrease the dimensionality of local feature descriptors, recent works propose several approaches to design binary, not real-valued, local feature descriptors, since they require much less storage. They also enable faster matching as there are efficient indexing schemes for binary vectors and Hamming distances can be computed fast on many architectures, including mobile devices.

In general, binary descriptors are composed of three parts: a sampling pattern, sampling pairs and orientation compensation. Precisely, the procedure or method to obtain each of these parts is different in each binary feature, as is shown in Table I. The descriptor is binary because it is composed out of ' 1 ' and ' 0 ' encoding the local information around the keypoint. Orientation compensation is obtained rotating the patch an determined angle and extracting a binary string describing the rotated patch. Both strings are no similar but the pairs remained the same. That ensures that the description is rotation invariant.

\section{[Table 1 about here.]}

Thanks to all these properties, binary descriptors are perfect candidates for real-time applications. BRIEF [28] uses random pre-determined locations, whereas BRISK [29] uses an exhaustive set of comparisons of close locations. ORB [30] relies on optimization and aims at improving the recognition rates by choosing the locations that decorrelate the tests. Similarly, FREAK [22] selects the intensity tests that provide the highest bit variance. Thanks to the simplicity of the above mentioned binary descriptors, they quickly attracted a lot of attention in the Computer Vision community and have become a very popular element of many real-time applications.

\subsection{Matching methods and strategies}

Once the features and their descriptors have been extracted from two or more images, they must be matched each other, i.e., all features in the image A are compared to all of the features in the image B in order to determine which corresponding locations in different images are the same feature. This strategy is the most basic and obvious choice and is called brute force 
matching. The feature descriptors can directly use Euclidean distances for descriptors represent the histogram of oriented gradient or the Hamming distance for binary descriptors, in feature space for ranking potential matches. For a Euclidean or Hamming distance give as metric, the simplest matching strategy is to set a threshold, i.e., maximum distance of similarity, and to return all matches from other images within this threshold. Too many false positives will be generated if the threshold is too high, i.e., incorrect matches will be returned. Too many false negatives will be generated if the threshold is too low, i.e., too many correct matches will be missed. A better strategy for this kind of brute-force method could be match the nearest neighbor in a cross check way too. A good match $(f a, f b)$ is obtained for images $I a$ and $I b$ if feature $f b$ is the best match for $f a$ in $I b$ and feature $f a$ is the best match for $f b$ in $I a$. In this way the matching only conserves the bidirectional matches and ideally the strongest correspondence has been kept. Ideally, this threshold itself will adapt to different regions of the feature space but requires additional time to calculate.

A second heuristic strategy can be to compare the nearest neighbor distance to that of the second nearest neighbor [31]. In order to filter the matches, Lowe [14] proposed to use a distance ratio test to try to eliminate false matches. The distance ratio between the two nearest matches of a considered keypoint is computed and it is a good match when this value is below a threshold. Indeed, this ratio allows helping to discriminate between ambiguous matches (distance ratio between the two nearest neighbors is close to one) and well discriminated matches. This nearest neighbor distance ratio can be defined as follows:

$$
\mathrm{NNDR}=\frac{d_{1}}{d_{2}}=\frac{\left\|D_{A}-D_{B}\right\|}{\left\|D_{A}-D_{C}\right\|}
$$

where $d_{1}$ and $d_{2}$ are the nearest and second nearest neighbor distances, $D_{A}$ is the target descriptor, and $D_{B}$ and $D_{C}$ are its closest two neighbors

So far, the simplest strategy to find all corresponding feature points is to match all features against all other features in each pair of images. Unfortunately, this brute force strategy is quadratic in the number of extracted features, which makes it impractical for most applications. A better strategy is to devise an indexing structure, such as a multi-dimensional search tree or a hash table, to rapidly search for features near a given feature. Multiindex hashing is a strategy [32] where, given a dataset populated with binary codes, each code is indexed $m$ times into $m$ different hash tables, according 
to $m$ adjacent substrings it has been divided into. Thus, given a query code, all the entries close to it at least in one substring are returned by search as neighbor candidates. Returned entries are then checked for validity by verifying that their full codes are not distant (in Hamming space) more than $r$ bits from query code. Another widely used class of indexing structures are multi-dimensional search trees. The best known of these are k-d trees, or kd-trees, which divide the multi-dimensional feature space along alternating axis-aligned hyperplanes, choosing the threshold along each axis so as to maximize some criterion, such as the search tree balance. The k-d tree recursively splits this plane along axis-aligned (horizontal or vertical) cutting planes. The splits are arranged so as to try to balance the tree, i.e., to keep its maximum depth as small as possible. Table II summarizes the method, strategy, norm used for matching, number of neighbours calculated and criterium of distance for all descriptors (HoGs based and binaries) used in this paper.

\subsection{Homography transformation}

Once some hypothetical (putative) matches have been obtained, geometric alignment can be used to verify which matches are inliers and which ones are outliers. A global geometric transform called homography can be fitted keeping only those feature matches (inliers) that are sufficiently close to this estimated transformation. It is possible to express this transformation in terms of matrix multiplication if we use homogeneous coordinates to express both the viewed point $\vec{Q}$ and the point $\vec{q}$ on the imager to which $\vec{Q}$ is mapped then we can express the action of the homography simply as $\vec{q}=s \cdot H \cdot \vec{Q}$, where the parameter $s$ is a scale factor and $H$ is a $3 \times 3$ matrix called homography matrix. The homography matrix $\mathrm{H}$ relates the positions of the points on a source image plane to the points on the destination image plane.

The process of selecting a small set of seed matches and then verifying a larger set is often called random sample consensus or RANSAC [33]. In the RANSAC method, subsets of the provided points are selected at random, and a homography matrix is computed for just that subset. It is then refined by all of the remaining data points that are roughly consistent with that initial estimation. The inliers are those that are consistent, while the outliers are those that are not. The RANSAC algorithm computes many such random samplings, and keeps the one that has the largest portion of inliers. This method is extremely efficient in practice for rejecting noisy outlier data and finding the correct answer. 
[Table 2 about here.]

\section{Results and discussion}

As an illustration of the possibilities of incorporating hardware nonidealities to the evaluation of the algorithm performance, we have chosen to test the influence of pixel-wise (pw) and column-wise FPN on the precision and recall rates of the matching for binary and non-binary local descriptors, specific SIFT, SURF, BRISK and ORB descriptors. As it will be explained below, precision is the fraction of true positive detections from all objects detected - including false positives - and recall is the fraction of true positive detections from all the relevant objects (keypoints) in the dataset, were they detected or not.

The images employed to test the algorithm have been taken from the Mikolajczyk benchmark [34] which is a publicly available and standard benchmark for evaluating local descriptors. The benchmark consists of 8 image sets, each containing 6 images that depict an increasing degree of a certain image transformation: i) Bark - zoom + rotation changes; ii) Bikes blur changes; iii) Boat - zoom + rotation changes; iv) Graffiti - view point changes; v) Leuven - illumination changes; vi) Trees - blur changes; vii) UBC - JPEG compression; viii) Wall - view point changes. This study has been focused on view point and rotation changes. Therefore the databases used have been the following: Bark, Boat, Graffiti and Wall. The matching score is computed on the test data, which is a set of images with a gradually increasing transformation between the reference frame and the other images. For example, for images sequence with viewpoint change, each image is rotated in 20 degrees with reference to the previous image. The ground truth is a homography that projects points to the reference frame. Figure 4 displays an example of an image of the dataset affected by growing values of both pixel-wise and column-wise FPN.

[Figure 4 about here.]

Fawcett [35] points that the performance of a matching algorithm at a particular threshold can be quantified by first counting the number of true and false matches and match failures, using the following definitions: predicted matches correctly detected $\left(T_{p}=\right.$ true positives); predicted no-matches incorrectly detected $\left(F_{n}=\right.$ false negatives); predicted matches incorrectly detected $\left(F_{p}=\right.$ false positives $) ;$ predicted no-matches correctly detected $\left(T_{n}=\right.$ 
true negatives) and then these numbers can be converted into unit rates: predicted matches $\left(P^{\prime}=T_{p}+F_{p}\right)$; predicted non-matches $\left(N^{\prime}=F_{n}+T_{n}\right)$; true matches $\left(P=T_{p}+F_{n}\right)$; true non-matches $\left(N=F_{p}+T_{n}\right)$; true positive rate or recall $\left(T P R=T_{p} / P\right)$; false positive rate $\left(F P R=F_{p} / N\right)$; positive predictive value or precision $\left(P P V=T_{p} / P^{\prime}\right)$; accuracy $\left(A C C=\left(T_{p}+T_{n}\right) /(P+N)\right)$. Figure 5 shows as sample the matching of SIFT, SURF, ORB and BRISK descriptors computed between the reference frame and an image with a viewpoint change of 40 degrees.

[Figure 5 about here.]

Figure 6 shows a sample confusion matrix or contingency table containing such numbers for a matching procedure based on cross checking and SIFT descriptor between the image one (or reference frame) and the image two (viewpoint $20^{\circ}$ ) in the database Graffiti with $0 \%$ of fixed pattern noise in both images. The matrix shows the number of true positives (TP), false positives (FP), false negatives (FN) and true negatives (TN). The columns sum up to the actual number of positives $(\mathrm{P})$ and negatives $(\mathrm{N})$, while the rows sum up to the predicted number of positives $\left(\mathrm{P}^{\prime}\right)$ and negatives $\left(\mathrm{N}^{\prime}\right)$. Besides, the positive predictive value (PPV) or precision, the negative predictive value (NPV), sensitivity or recall (class) or true positive rate (TPR), the false positive rate (FPR), the false negative rate (FNR), the specificity or true negative rate (TNR), and the accuracy (ACC) are shown.

[Figure 6 about here.]

The ideal system corresponds to precision and recall equal to one. In practice, a compromise between these two quantities exists: a system with a high recall is likely to have false positives, and a system with high precision is likely to miss some true annotations. Often, the two quantities are summarized into a single number, $F$, defined as the harmonic mean of precision $(P)$ and recall $(R): F=2 P R / P+R$. The value of $\mathrm{F}$ measures the accuracy of a test that presents a binary classification, weighting precision $(P)$ and completeness or recall $(R)$. Take values from 0 to 1 with 1 being the best possible case and 0 the worst case. The harmonic mean between precision and recall has been considered, known as traditional F-Measure or balanced F1Score.

The consequences of applying different values for the mismatch in the threshold of the pixels' source follower, which results in a pw-FPN, and the 
columns' bias current, that results in a cw-FPN, can be seen in Figures 7, 8, 9 and 10; and Figures 11, 12, 13 and 14, respectively. Therefore, cw-FPN is varied for a fixed value of the pw-FPN in Figures 7 to 10 and, and the other way around in Figures 11 to 14. Within each graph, each line corresponds to a F1Score for a descriptor, a method of matching and a change of viewpoint against a percent of noise — which ranges from $0 \%$ to $100 \%$ in steps of $5 \%$ The figures below show the matching score of different detectors measured across viewpoint angle. Each point on a curve is the matching score computed between the reference frame and image with a viewpoint change with a percent of PFN noise.

Observing the graphs, it can be clearly seen that the databases contaminated with cw-FPN undergoing only a change of viewpoint have a higher F1Score and decay with a lower slope than those databases that also have zoom. From these previous databases, it can be seen that between 40 and $60 \%$ of the cw-PFN noise, F1Score has a value of 0 for all descriptors and matching methods. Obviously it can also be observed that, as the angle changing the view and zoom is greater between the reference image and the rest of the images, the F1Score is smaller. It also seems that the FLANN and brute force methods work better with low noise whereas the cross checking method works better with high noise level.

[Figure 7 about here.]

[Figure 8 about here.]

[Figure 9 about here.]

[Figure 10 about here.]

[Figure 11 about here.]

[Figure 12 about here.]

[Figure 13 about here.]

[Figure 14 about here.] 
It can be seen that for a zero fixed value of cw-FPN, by increasing the value of pw-FPN - what can be seen in Figure 11 to Figure 14 plots -, the degradation of F1Score is much weaker than the variation obtained by fixing pw-FPN to zero and increasing cw-FPN — which is depicted in Figure 7 to Figure 10 plots. It can be concluded that cw-FPN has a stronger deteriorating effect in the object detection algorithm than pw-FPN has. This is specially important in CMOS imagers which include column-parallel ADC readout architectures. Therefore, given a feature extraction algorithm and a specified accuracy, these graphs provide a method to define the maximum cw-FPN allowed when designing the CMOS ADC. This allows to relax the ADC specifications in order to find a balance between circuit power dissipation and algorithm accuracy.

Equivalently, from the point of view of the algorithm, a new cascade of classifiers could be trained to cope with the artifacts caused by a large cw-FPN. Either way, the influence of hardware non-idealities are evidenced with this methodology, allowing comprehensive optimization and co-design of image sensor hardware and algorithm parameters.

\section{Conclusions and future work}

The design of an embedded vision system cannot always be achieved through a traditional top-down approach. Optimization at different levels is necessary. Thus, it is possible to work at algorithm levels and incorporate a low level description of the non-idealities of the image sensor to evaluate its influence on the performance of the vision algorithm. A feasible way to do this is to incorporate the necessary models that allows to evaluate the effect of the deviation of the physical parameters of the sensor with the performance of the artificial vision algorithms and their implementation. This has been done in this work. The main advantage is that these models are well known by the community of vision application developers. The co-design of vision hardware and software is possible in this way. As an example, we have shown the effect of pw- and cw-FPN in the detection and description of local features.

Nonetheless, there is much scope for extension. In particular, a more sophisticated means of obtaining the number of matches correctly and incorrectly estimated by a feature matching algorithm is desirable. This would likely require more fundamental research in the field. On the other hand, the complete imaging methodology could be enhanced by also incorporating 
other design effects (i.e., optical design). Nevertheless, the study in this direction is beyond the scope of this paper and left for the subject of our next comparison.

\section{Acknowledgements}

The author wants to acknowledge the work of the rest of the participants in this project, namely: J.A. López-Alcantud, P. Rubio-Ibáñez, Universidad Politécnica de Cartagena, J. A. Díaz-Madrid, Centro Universitario de la Defensa-UPCT and T. J. Kazmierski, University of Southampton.

This work was mainly supported by the Spanish MINECO and the European Region Development Fund (ERDF/FEDER) through project 'iCaveats' (Ref. TEC2015-66878-C3-1-R and TEC2015- 66878-C3-2-R), and partially supported by Junta de Andalucía through project 'SmartCIS3D' (Ref. TIC 2338-2013), by the Consellería de Cultura, Educ. e Ord. Universitaria and ERDF (accreditation 2016-2019, ED431G/08 and reference competitive group 2017-2020, ED431C 2017/69) and by EU-REA through project 'Achieve' (EU H2020 MSCA- ITN 2017, Grant No. 765866).

[1] E. R. Fossum, CMOS image sensors: electronic camera on a chip, in: Proceedings of International Electron Devices Meeting, ISSN 0163-1918, 17-25, doi:\let\@tempa\bibinfo@X@doi10.1109/ IEDM.1995.497174, 1995.

[2] H. Tian, B. Fowler, A. E. Gamal, Analysis of Temporal Noise in CMOS Photodiode Active Pixel Sensor, 2001.

[3] J. Nakamura, Image Sensors and Signal Processing for Digital Still Cameras, CRC Press, Inc., Boca Raton, FL, USA, ISBN 0849335450, 2005.

[4] K. Matsumoto, T. Nakamura, A. Yusa, S. Nagai, A New MOS Phototransistor Operating in a Non-Destructive Readout Mode, Japanese Journal of Applied Physics 24 (5A) (1985) L323, URL http://stacks . iop.org/1347-4065/24/i=5A/a=L323.

[5] E. R. Fossum, Active pixel sensors: are CCDs dinosaurs?, doi:\let @tempa \bibinfo@X@doi10.1117/12.148585, URL https://doi.org/ 10.1117/12.148585, 1993. 
[6] D. A. George, I. D. Andrew, E. E. sayed Ibrarhim, Active Pixel Sensor And Imaging System Having Differential Mode, URL https://lens. org/099-895-948-098-172, ????

[7] H. Zimmermann, Integrated Silicon Optoelectronics, Physics and astronomy online library, Springer Berlin Heidelberg, ISBN 9783540666622, URL https://books . google.es/books?id=maRCv9SQfowC, 2000.

[8] C. L. T, B. M. A, H. E. J, Sensor Cell Having A Soft Saturation Circuit, URL https://lens.org/022-956-747-444-748, ????

[9] J. Ohta, Smart CMOS image sensors and applications, CRC press, 2007.

[10] C. Villegas-Pachón, R. Carmona-Galán, J. Fernández-Berni, Á. Rodríguez-Vázquez, Hardware-aware performance evaluation for the co-design of image sensors and vision algorithms, in: 2016 13th International Conference on Synthesis, Modeling, Analysis and Simulation Methods and Applications to Circuit Design (SMACD), 1-4, doi:〈let \@tempa \bibinfo@X@doi10.1109/SMACD.2016.7520722, 2016 .

[11] F.-B. Jorge, C.-G. Ricardo, R. Rocío, R.-V. Angel, Bottom-up performance analysis of focal-plane mixed-signal hardware for Viola-Jones early vision tasks, International Journal of Circuit Theory and Applications 43 (8) (????) 1063-1079, doi: \let\@tempa\bibinfo@X@ doi10.1002/cta.1996, URL https://onlinelibrary.wiley.com/doi/ abs/10.1002/cta.1996.

[12] B. Jähne, EMVA 128 Standard for Machine Vision - Objective Specification of Vital Camera Data, Optik \& Photonik 5 (2010) 53-54, doi: $\backslash$ let\@tempa \bibinfo@X@doi10.1002/opph.201190082.

[13] Official Website of the EMVA1288 Standard, http://www. standard1288.org/, accessed: 2018-06-30, ????

[14] D. G. Lowe, Distinctive Image Features from Scale-Invariant Keypoints, International Journal of Computer Vision 60 (2004) 91-110.

[15] H. Bay, T. Tuytelaars, L. V. Gool, Surf: Speeded up robust features, in: In ECCV, 404-417, 2006. 
[16] K. Simonyan, A. Vedaldi, A. Zisserman, Descriptor Learning Using Convex Optimisation, in: A. Fitzgibbon, S. Lazebnik, P. Perona, Y. Sato, C. Schmid (Eds.), Computer Vision - ECCV 2012, Springer Berlin Heidelberg, Berlin, Heidelberg, ISBN 978-3-642-33718-5, 243-256, 2012.

[17] E. Tola, V. Lepetit, P. Fua, A fast local descriptor for dense matching .

[18] S. Leutenegger, M. Chli, R. Siegwart, BRISK: Binary Robust invariant scalable keypoints., in: D. N. Metaxas, L. Quan, A. Sanfeliu, L. J. V. Gool (Eds.), ICCV, IEEE Computer Society, ISBN 978-1-45771101-5, 2548-2555, URL http://dblp.uni-trier.de/db/conf/iccv/ iccv2011.html\#LeuteneggerCS11, 2011.

[19] M. Calonder, V. Lepetit, C. Strecha, P. Fua, BRIEF: Binary Robust Independent Elementary Features, in: K. Daniilidis, P. Maragos, N. Paragios (Eds.), Computer Vision - ECCV 2010, Springer Berlin Heidelberg, Berlin, Heidelberg, ISBN 978-3-642-15561-1, 778-792, 2010.

[20] E. Rublee, V. Rabaud, K. Konolige, G. Bradski, ORB: an efficient alternative to SIFT or SURF, in: In ICCV, ????

[21] G. Levi, T. Hassner, LATCH: Learned Arrangements of Three Patch Codes, CoRR abs/1501.03719, URL http://arxiv.org/abs/1501. 03719.

[22] A. Alahi, R. Ortiz, P. Vandergheynst, FREAK: Fast Retina Keypoint., in: CVPR, IEEE Computer Society, ISBN 978-1-4673-1226-4, 510-517, URL http://dblp.uni-trier.de/db/conf/cvpr/cvpr2012. html\#AlahiOV12, 2012.

[23] P. F. Alcantarilla, A. Bartoli, A. J. Davison, KAZE Features, in: Proceedings of the 12th European Conference on Computer Vision - Volume Part VI, ECCV'12, Springer-Verlag, Berlin, Heidelberg, ISBN 978-3-642-33782-6, 214-227, doi: \let\@tempa \bibinfo@X@ doi10.1007/978-3-642-33783-3_16, URL http://dx.doi.org/10.1007/ 978-3-642-33783-3_16, 2012.

[24] A. B. Pablo Alcantarilla (Georgia Institute of Technolog), Jesus Nuevo (TrueVision Solutions AU), Fast Explicit Diffusion for Accelerated Features in Nonlinear Scale Spaces, in: Proceedings of the British Machine Vision Conference, BMVA Press, 2013. 
[25] M. J. M. Pelgrom, A. C. J. Duinmaijer, A. P. G. Welbers, Matching properties of MOS transistors, IEEE Journal of Solid-State Circuits 24 (5) (1989) 1433-1439, ISSN 0018-9200, doi: \let\@tempa\bibinfo@ X@doi10.1109/JSSC.1989.572629.

[26] C. Harris, M. Stephens, A combined corner and edge detector, in: In Proc. of Fourth Alvey Vision Conference, 147-151, 1988.

[27] H. Bay, A. Ess, T. Tuytelaars, L. V. Gool, B. K. U. Leuven, Speeded-Up Robust Features (SURF), 2008.

[28] M. Calonder, V. Lepetit, M. Ozuysal, T. Trzcinski, C. Strecha, P. Fua, BRIEF: Computing a Local Binary Descriptor Very Fast, IEEE Transactions on Pattern Analysis and Machine Intelligence 34 (7) (2012) 1281-1298, ISSN 0162-8828, doi:\let\@tempa \bibinfo@X@doi10.1109/ TPAMI.2011.222.

[29] S. Leutenegger, M. Chli, Y. Siegwart, Brisk: Binary robust invariant scalable keypoints, in: In Computer Vision (ICCV), 2011 IEEE International Conference on, 2548-2555, 2011.

[30] E. Rublee, V. Rabaud, K. Konolige, G. Bradski, ORB: An Efficient Alternative to SIFT or SURF, in: Proceedings of the 2011 International Conference on Computer Vision, ICCV '11, IEEE Computer Society, Washington, DC, USA, ISBN 978-1-4577-1101-5, 2564-2571, doi: \let\@tempa \bibinfo@X@doi10.1109/ICCV.2011.6126544, URL http: //dx.doi.org/10.1109/ICCV.2011.6126544, 2011.

[31] M. Brown, D. Lowe, Invariant Features from Interest Point Groups, in: In British Machine Vision Conference, 656-665, 2002.

[32] M. Norouzi, A. Punjani, D. J. Fleet, Fast search in Hamming space with multi-index hashing, in: 2012 IEEE Conference on Computer Vision and Pattern Recognition, ISSN 1063-6919, 3108-3115, doi:〈let \ @tempa \bibinfo@X@doi10.1109/CVPR.2012.6248043, 2012.

[33] M. A. Fischler, R. C. Bolles, Random Sample Consensus: A Paradigm for Model Fitting with Applications to Image Analysis and Automated Cartography, Communications of the ACM 24 (6) (1981) 381-395. 
604

[34] K. Mikolajczyk, T. Tuytelaars, C. Schmid, A. Zisserman, J. Matas, F. Schaffalitzky, T. Kadir, L. Van Gool, A Comparison of Affine Region Detectors 65 (2005) 43-72.

[35] T. Fawcett, An introduction to ROC analysis, Pattern Recognition Letters 27 (8) (2006) 861-874, ISSN 01678655, doi:\let @tempa \bibinfo@X@doi10.1016/j.patrec.2005.10.010, URL http:// dx.doi.org/10.1016/j.patrec.2005.10.010. 


\begin{tabular}{llll}
\hline & Sampling pattern & $\begin{array}{l}\text { Orientation calcu- } \\
\text { lation }\end{array}$ & Sampling pairs \\
\hline BRIEF & None. & None. & Random. \\
ORB & None. & Moments. & Learned pairs. \\
BRISK & Concentric circles & Comparing gradi- & Using only short \\
& with more points & ents of long pairs. & pairs. \\
FREAK & $\begin{array}{l}\text { On outer rings. } \\
\text { Centric circles with }\end{array}$ & ents of preselected & \\
& $\begin{array}{l}\text { more points on } \\
\text { inner rings. }\end{array}$ & & \\
& & & \\
\hline
\end{tabular}

Table I: Method elected for each part of a binary descriptor 


\begin{tabular}{|c|c|c|c|c|c|}
\hline Method & Strategy & Descriptor & Norm & $\mathrm{K}$ & Threshold \\
\hline \multirow{2}{*}{\multicolumn{2}{|c|}{$\begin{array}{l}\text { Cross Checkin } \\
\text { Brute Force }\end{array}$}} & Binary & Hamming & 1 & $\begin{array}{l}4 \times \min . \text { dis- } \\
\text { tance. }\end{array}$ \\
\hline & & non-Binary & Euclidean & 1 & $\begin{array}{l}4 \times \min . \text { dis- } \\
\text { tance. }\end{array}$ \\
\hline \multirow{4}{*}{ FLANN } & KNN & Binary & Hamming & 2 & $\mathrm{NNDR}=0.78$ \\
\hline & MNIV & non-Binary & Euclidean & 2 & $\mathrm{NNDR}=0.78$ \\
\hline & $\begin{array}{ll}\text { LSH } & \text { In- } \\
\operatorname{dex} / \mathrm{KNN}\end{array}$ & Binary & Hamming & 2 & $\mathrm{NNDR}=0.78$ \\
\hline & K-D Tree/KNN & non-Binary & Euclidean & 2 & $\mathrm{NNDR}=0.78$ \\
\hline
\end{tabular}

Table II: Methods and strategies elected for matching of binaries and a HOG-based descriptors. 


\section{List of Figures}

13 Active pixel sensor and read-out electronics . . . . . . . . . 25

2 Mathematical model of a single pixel. Usually, $K, \eta$ and $\mu_{d}$ are unknown model parameters. $\mu$ and $\sigma^{2}$ represents the mean and variance of the number of photons. . . . . . . . . . . . 26

3 Methodological flow: a certain percentage of fixed pattern noise is added to a query image and to a training image, respectively; the keypoints are obtained by means of different descriptors (binary and non-binary), matching is obtained in order to get positive (good) matches by means of brute force with NNDR, cross-checking or using FLANN; the homography is used as truth ground to obtain true positives (inliers) and false positives (outliers) using the RANSAC algorithm. . . 27

4 Output images for growing values of (up) cw-FPN and (bottom) pw-FPN which ranges from $0 \%$ to $100 \%$ in steps of $5 \%$ with respect to the signal range for the reference image in database Graffiti. . . . . . . . . . . . . . . . . . . . . . . . . . 28

$5 \quad$ Image matching with brute force between reference image (query image) and image with a view point of view of 40 degrees (training image) for (upper to bottom) SIFT, SURF, ORB and BRISK descriptors with a noise percent of $\mathrm{cw}-\mathrm{FPN}=30 \%$ and pw-FPN $=0 \%$ in both images. Left, reference image and image with a view point of view of 40 degrees showing keypoints detected. Right, homography transformation based on keypoints detected in both images showing inliers keypoints $\left(T_{p}\right)$ used for transformation. . . . . . . . . . . . . . . . 29

6 Confusion matrix shows the number of matches correctly and incorrectly estimated by a feature matching algorithm. . . . . 30

7 F1Score vs. cw-FPN for four databases (Graffiti, Bark, Wall and Boat): for SIFT . . . . . . . . . . . . 31

8 F1Score vs. cw-FPN for four databases (Graffiti, Bark, Wall and Boat) for SURF . . . . . . . . . . . . . . 32

9 F1Score vs. cw-FPN for four databases (Graffiti, Bark, Wall and Boat): for ORB . . . . . . . . . . . . . . 33

10 F1Score vs. cw-FPN for four databases (Graffiti, Bark, Wall and Boat): for BRISK . . . . . . . . . . . . . 34 
647

648

649

650

651

652

653

654

11 F1Score vs. pw-FPN for four databases (Graffiti, Bark, Wall and Boat) for SIFT . . . . . . . . . . . . . . 35

12 F1Score vs. pw-FPN for four databases (Graffiti, Bark, Wall and Boat) for SURF . . . . . . . . . . . . . 36

13 F1Score vs. pw-FPN for four databases (Graffiti, Bark, Wall and Boat) for ORB ................ . . . 37

14 F1Score vs. pw-FPN for four databases (Graffiti, Bark, Wall and Boat) for BRISK . . . . . . . . . . . . 38 


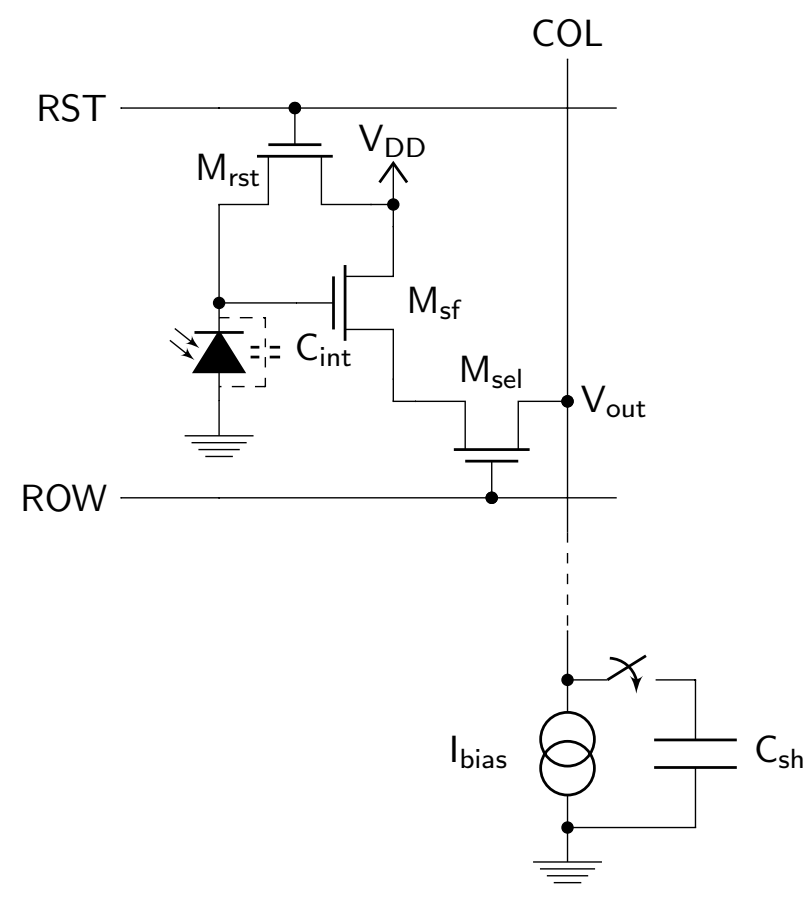

Figure 1 


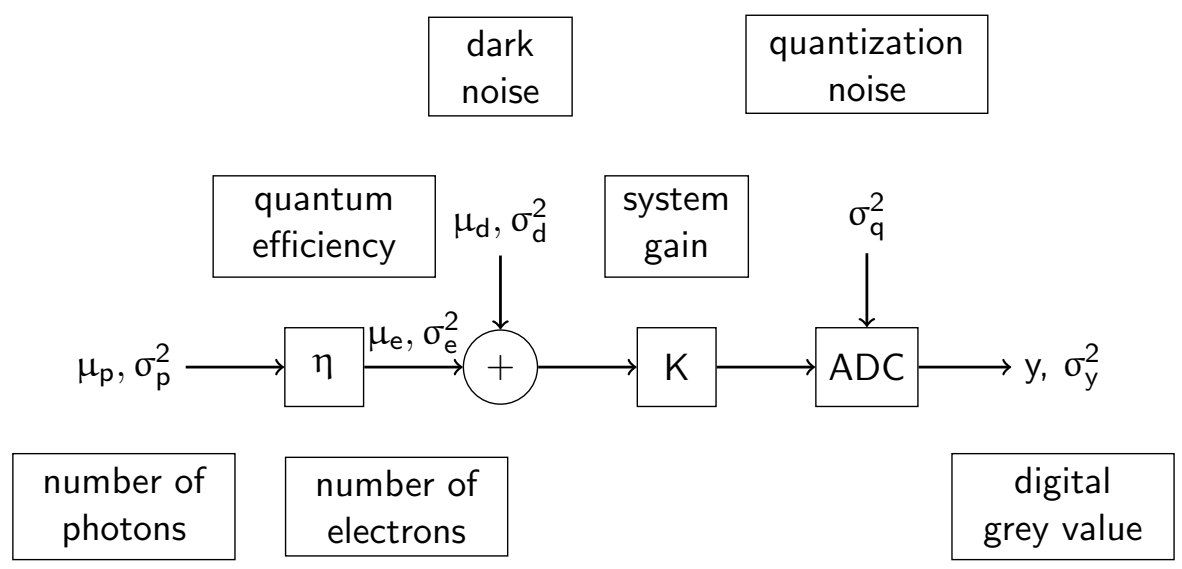

Figure 2 


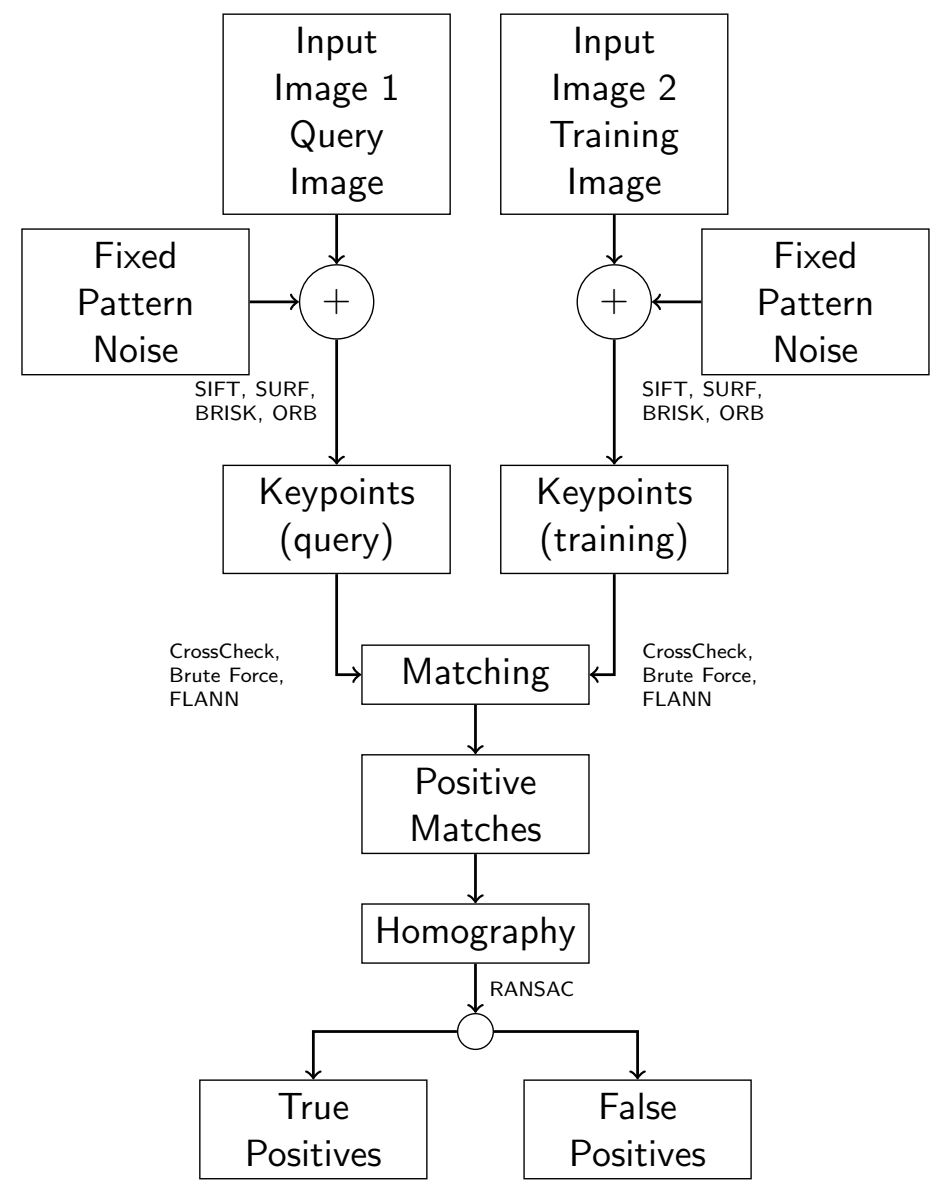

Figure 3 

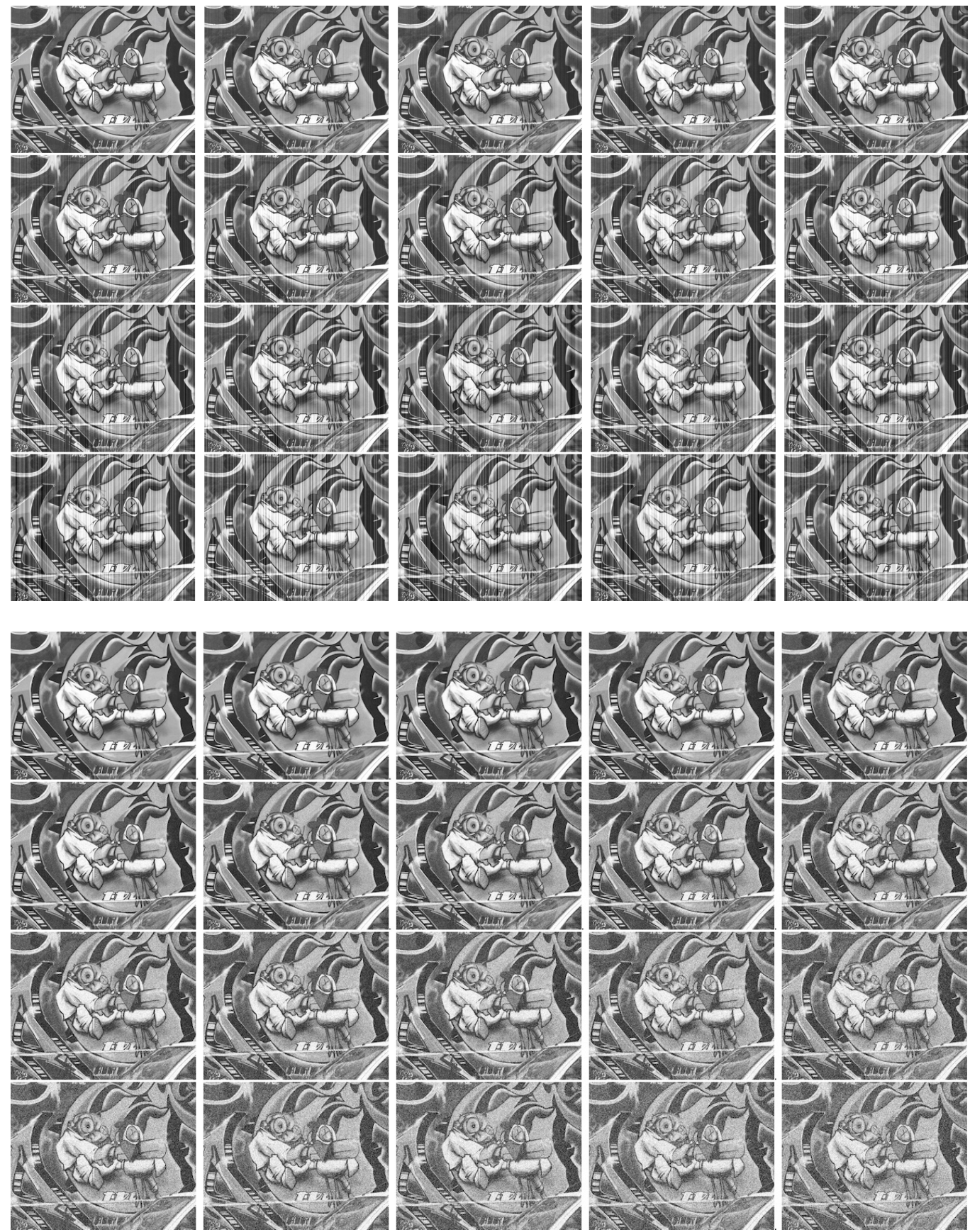

Figure 4 


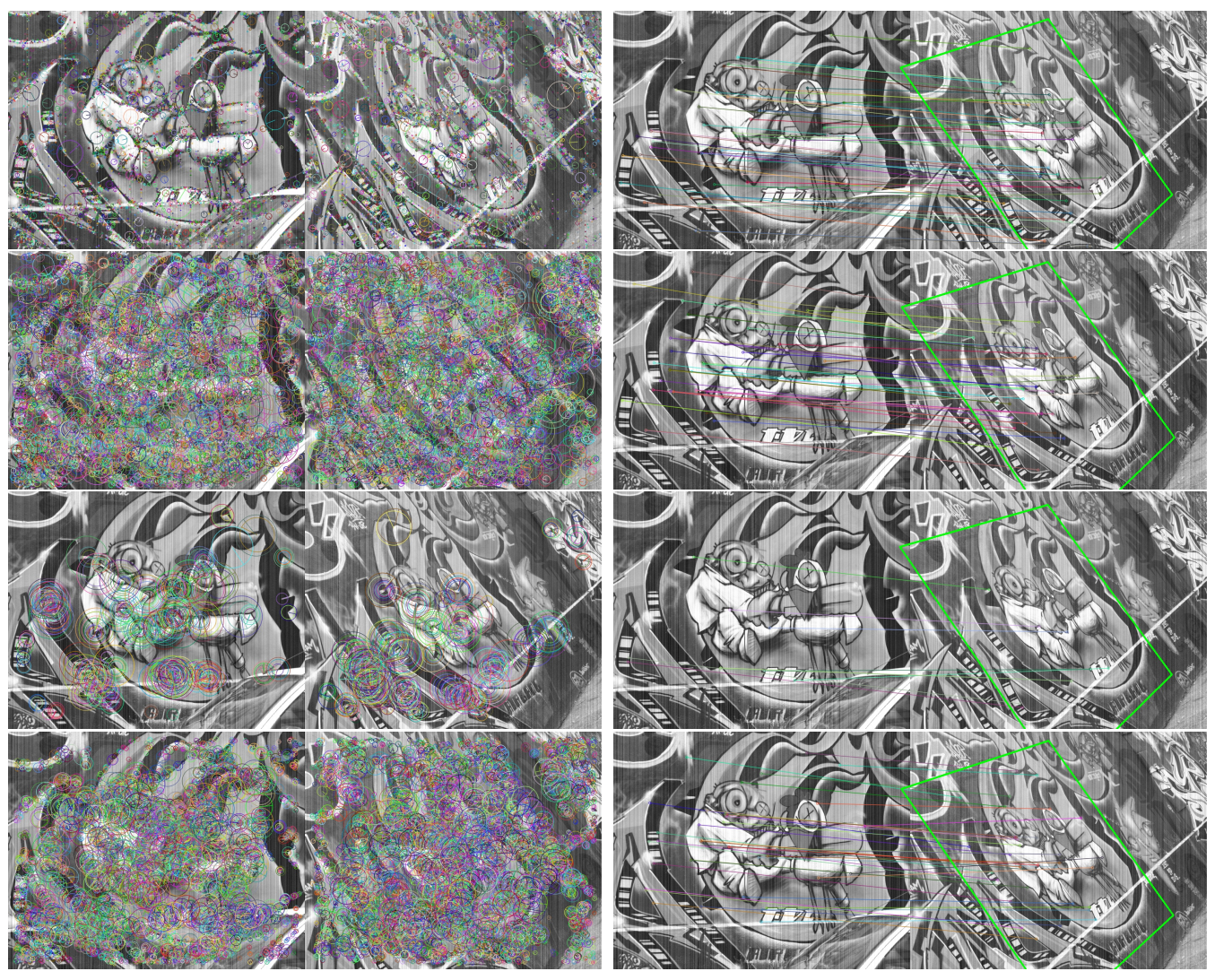

Figure 5 


\begin{tabular}{|c|c|c|c|c|}
\hline \multirow{2}{*}{ Pred. Matches } & True Matches & \multicolumn{2}{|c|}{ True non-Matches } & \\
\hline & $T_{p}=995$ & $F_{p}=156$ & $P^{\prime}=1151$ & $\begin{array}{c}P P V= \\
0.8644\end{array}$ \\
\hline \multirow[t]{4}{*}{ Pred. non-Matches } & $F_{n}=1802$ & $T_{n}=2099$ & $\mathrm{~N}^{\prime}=3901$ & $\begin{array}{c}N P V= \\
0.5380\end{array}$ \\
\hline & $P=2797$ & $N=2255$ & $\mathrm{~T}=5052$ & \\
\hline & $\begin{array}{c}T P R= \\
0.3557\end{array}$ & $\begin{array}{c}T N R= \\
0.9433\end{array}$ & & $\begin{array}{c}A C C= \\
0.6124\end{array}$ \\
\hline & $\begin{array}{c}F N R= \\
0.6443\end{array}$ & $\begin{array}{c}F P R= \\
0.0567\end{array}$ & & \\
\hline
\end{tabular}

Figure 6 


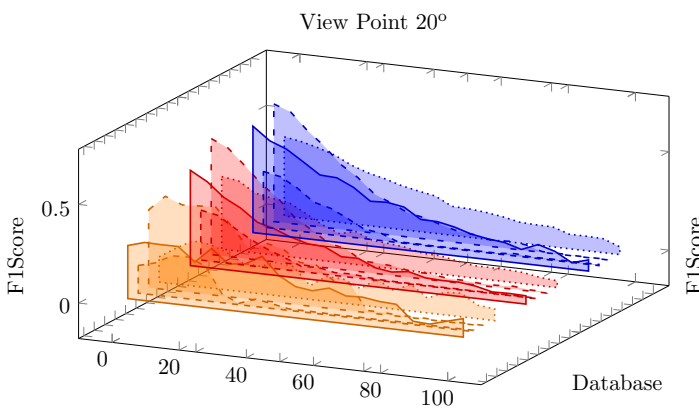

Percentage of cw-FPN

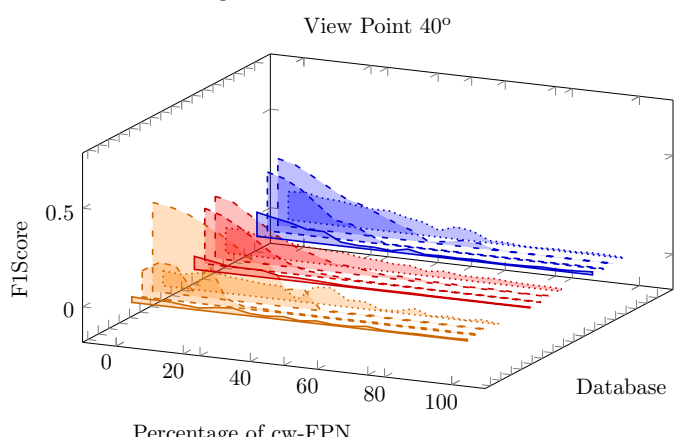

Percentage of cw-FPN



Percentage of cw-FPN

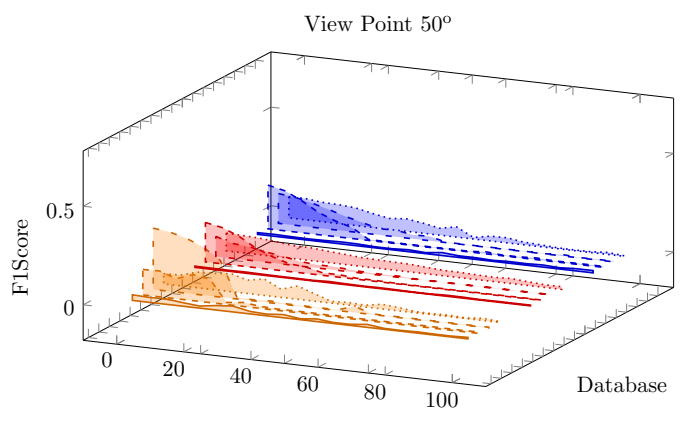

Percentage of cw-FPN

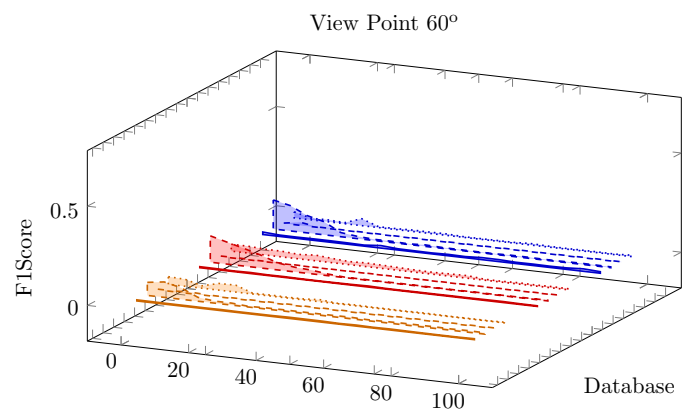

Percentage of cw-FPN

Cross Check: — GRAF - - - BARK - - WALL …… BOAT
Brute Force: — GRAF - - BARK - - WALL …… BOAT
FLANN: - GRAF - - BARK - - WALL …… BOAT

Figure 7 




Percentage of cw-FPN

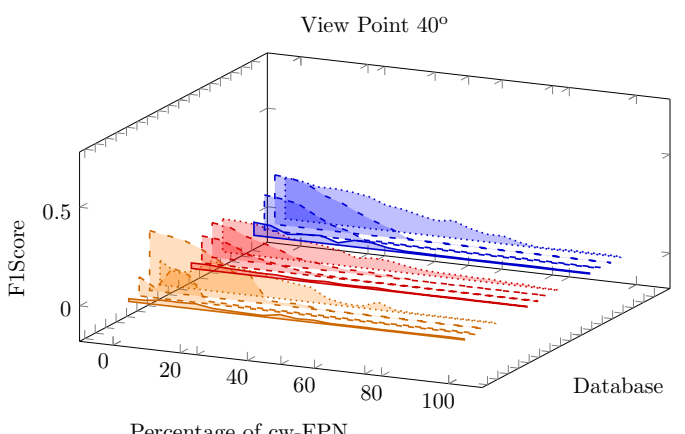

Percentage of cw-FPN

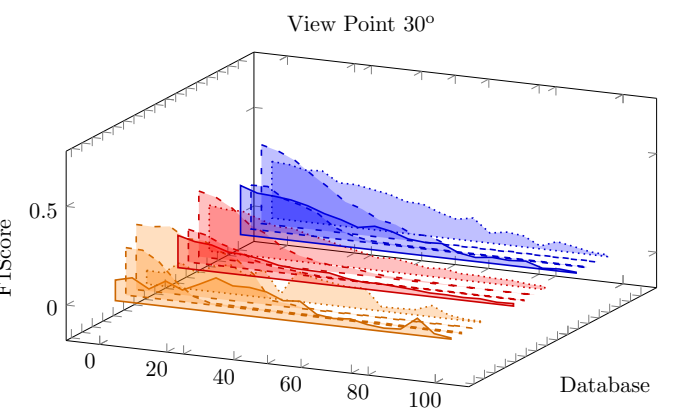

Percentage of cw-FPN

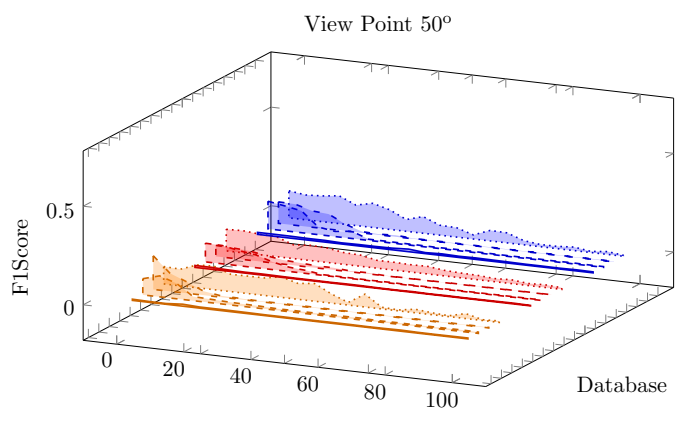

Percentage of cw-FPN

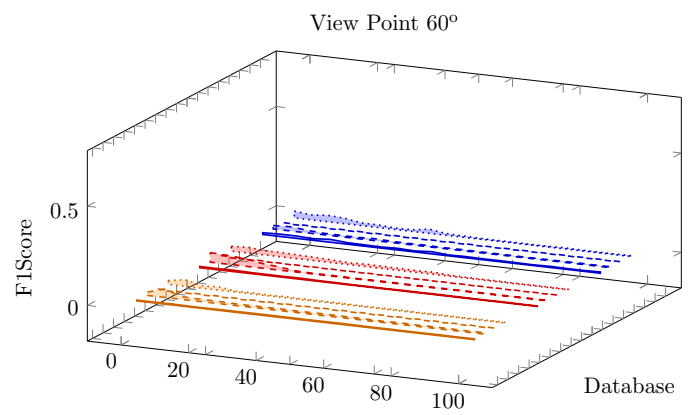

Percentage of cw-FPN

Cross Check: — GRAF - - - BARK - - WALL …… BOAT
Brute Force: — GRAF - - BARK - - WALL …… BOAT
FLANN: - GRAF - - BARK - - WALL …… BOAT

Figure 8 


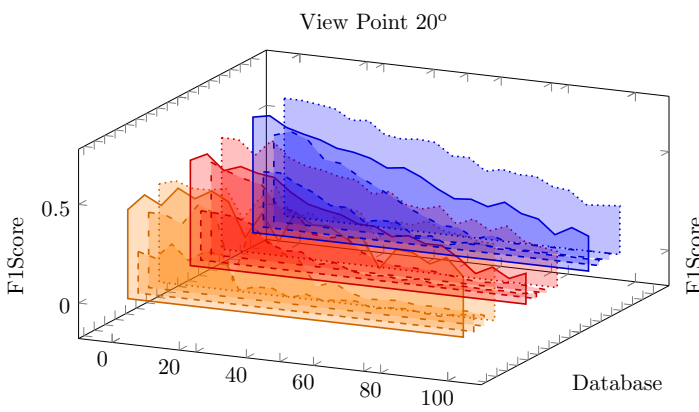

Percentage of cw-FPN



Percentage of cw-FPN



Percentage of cw-FPN



Percentage of cw-FPN

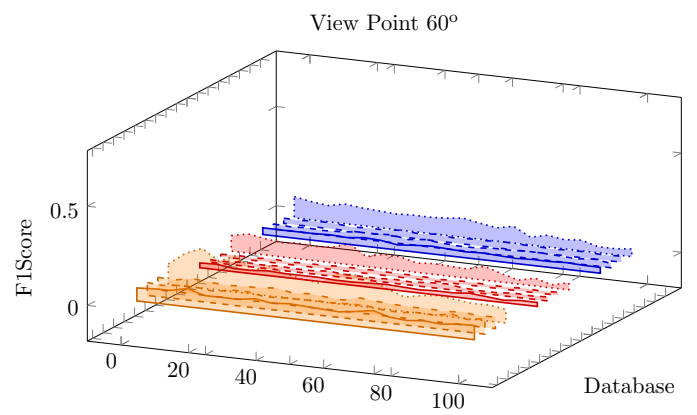

Percentage of cw-FPN

Cross Check: — GRAF - - - BARK - - WALL …… BOAT
Brute Force: — GRAF - - BARK - - WALL …… BOAT
FLANN: - GRAF - - BARK - - WALL …… BOAT

Figure 9 




Percentage of cw-FPN



Percentage of cw-FPN

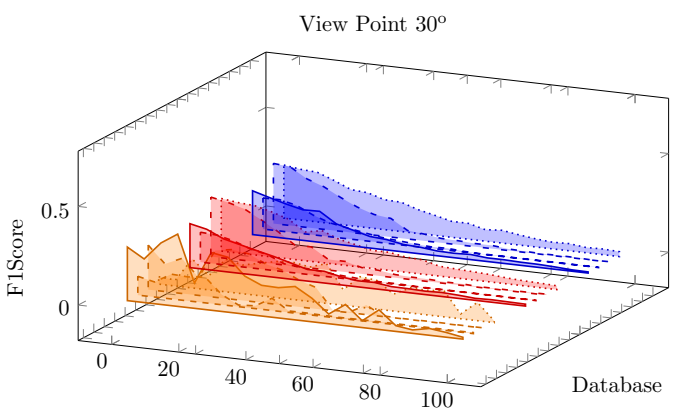

Percentage of cw-FPN

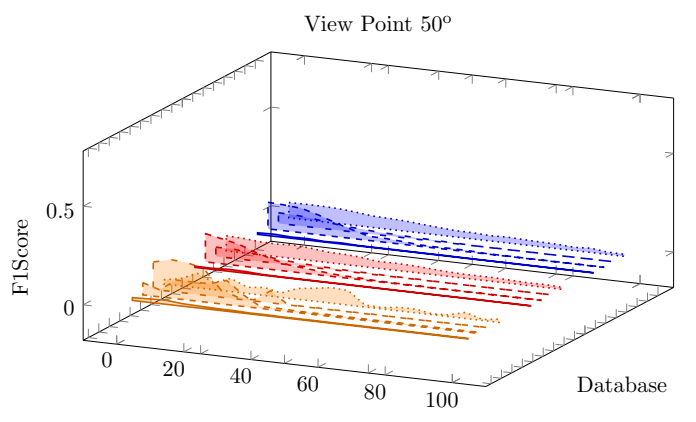

Percentage of cw-FPN

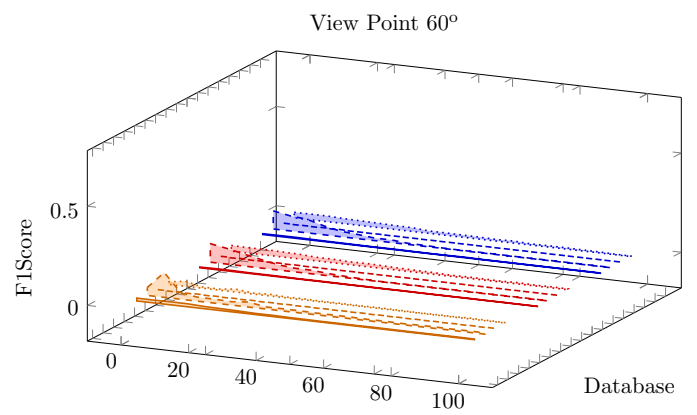

Percentage of cw-FPN

Cross Check: — GRAF - - - BARK - - WALL …… BOAT
Brute Force: — GRAF - - BARK - - WALL …… BOAT
FLANN: - GRAF - - BARK - - WALL …… BOAT

Figure 10 


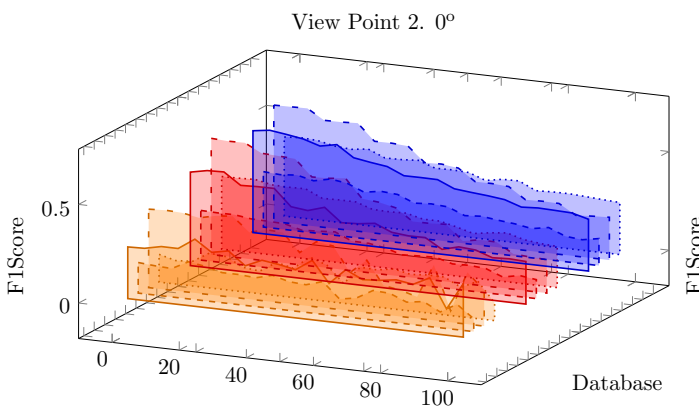

Percentage of pw-FPN

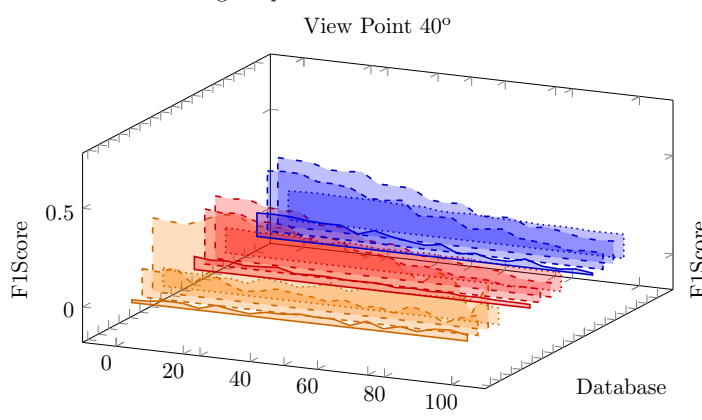

Percentage of pw-FPN

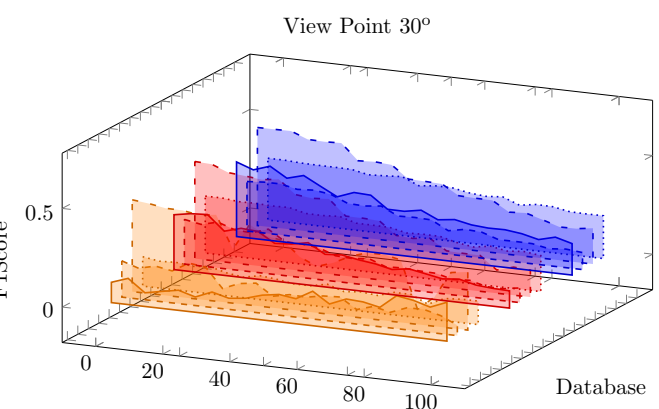

Percentage of pw-FPN



Percentage of pw-FPN

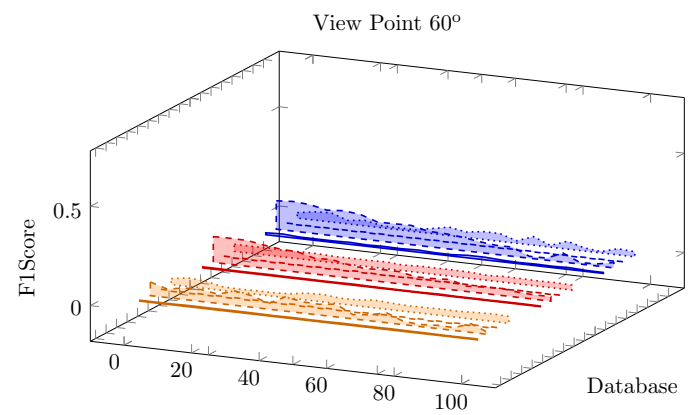

Percentage of pw-FPN

\begin{tabular}{l}
\hline Cross Check: — GRAF - - - BARK - - WALL …… BOAT \\
Brute Force: — GRAF - - B BARK - - WALL …… BOAT \\
FLANN: — GRAF - - BARK - - WALL …… BOAT
\end{tabular}

Figure 11 


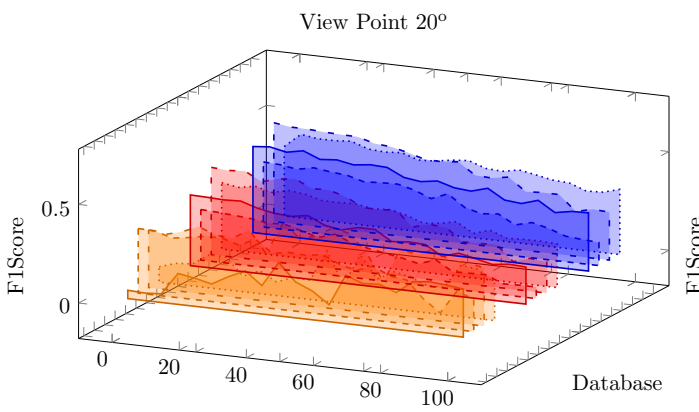

Percentage of pw-FPN

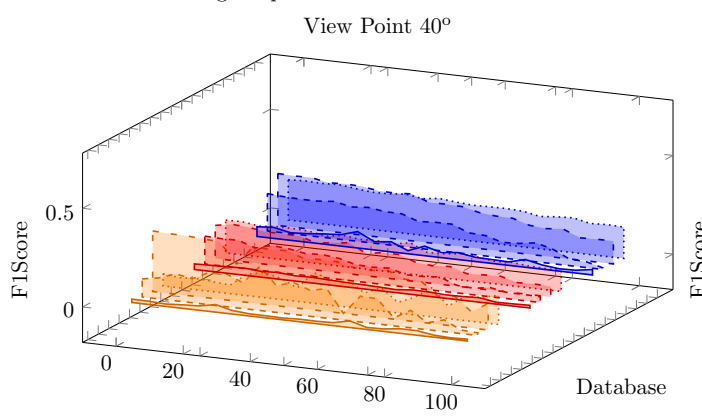

Percentage of pw-FPN

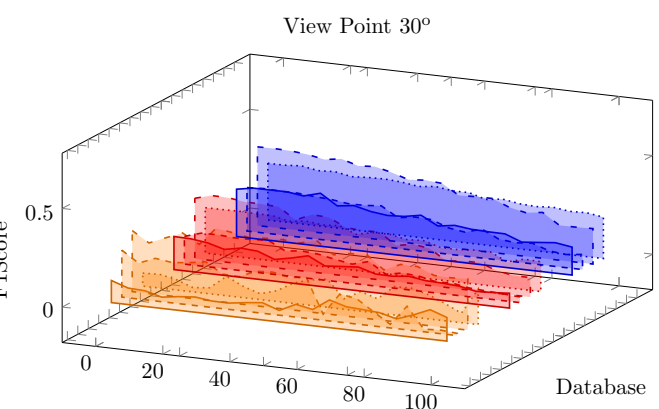

Percentage of pw-FPN

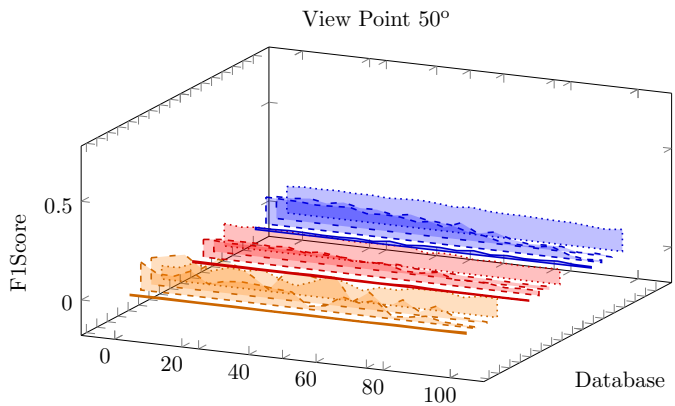

Percentage of pw-FPN

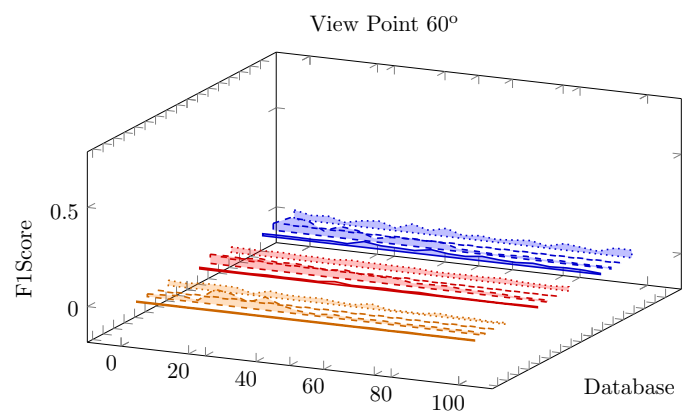

Percentage of pw-FPN

\begin{tabular}{l}
\hline Cross Check: — GRAF - - - BARK - - WALL …… BOAT \\
Brute Force: — GRAF - - B BARK - - WALL …… BOAT \\
FLANN: — GRAF - - BARK - - WALL …… BOAT
\end{tabular}

Figure 12 


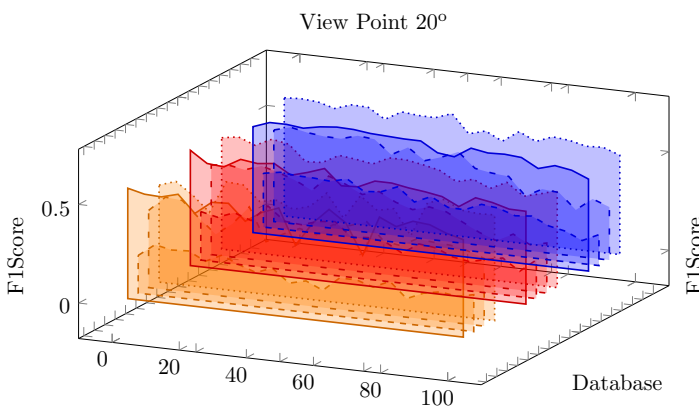

Percentage of pw-FPN

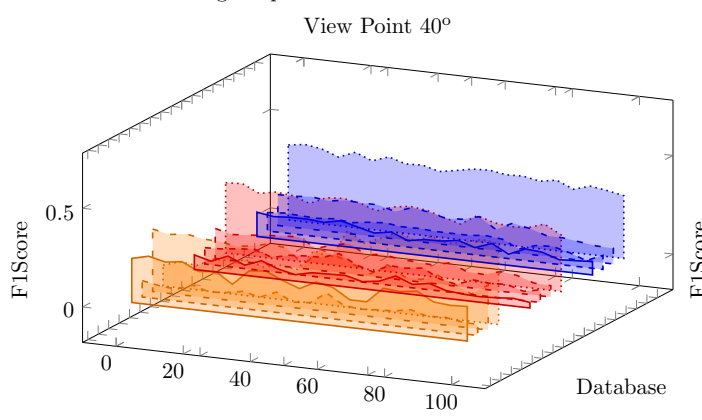

Percentage of pw-FPN

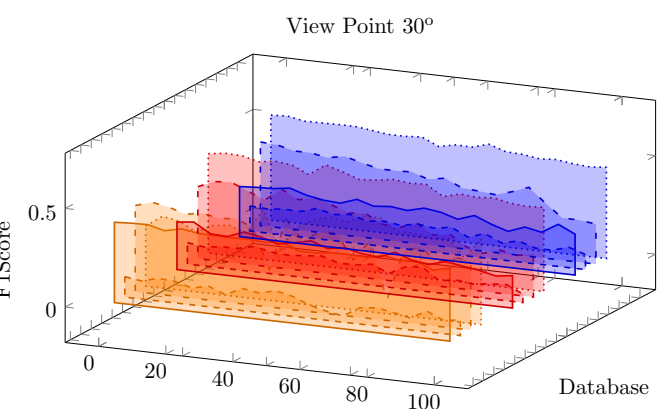

Percentage of pw-FPN

View Point $50^{\circ}$



Percentage of pw-FPN

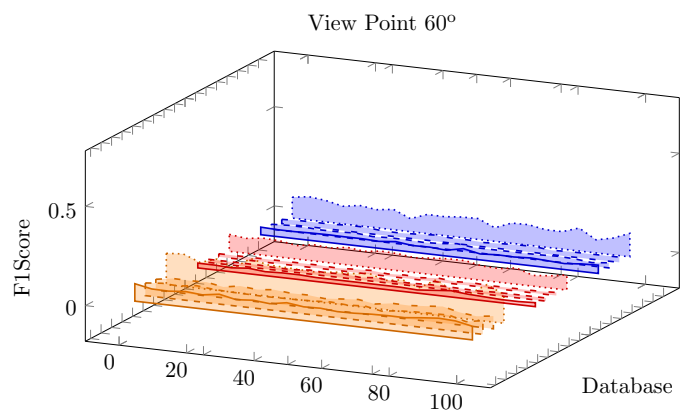

Percentage of pw-FPN

\begin{tabular}{l}
\hline Cross Check: — GRAF - - - BARK - - WALL …… BOAT \\
Brute Force: — GRAF - - B BARK - - WALL …… BOAT \\
FLANN: — GRAF - - BARK - - WALL …… BOAT
\end{tabular}

Figure 13 




Percentage of pw-FPN

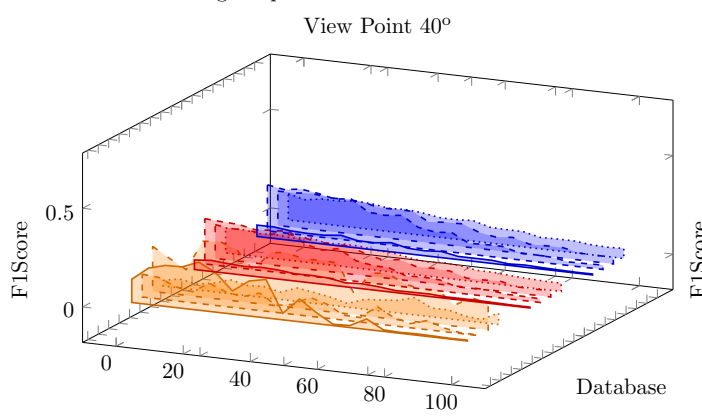

Percentage of pw-FPN

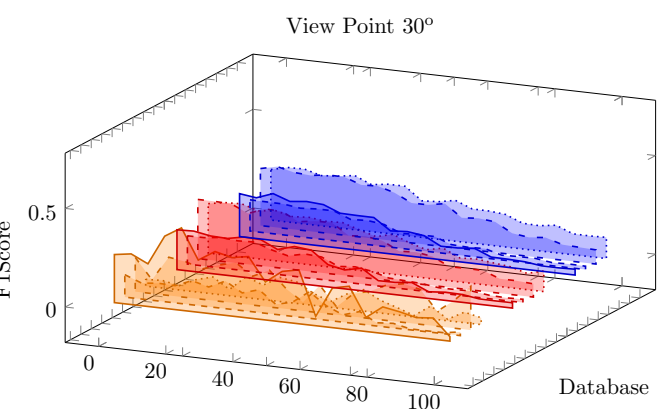

Percentage of pw-FPN



Percentage of pw-FPN

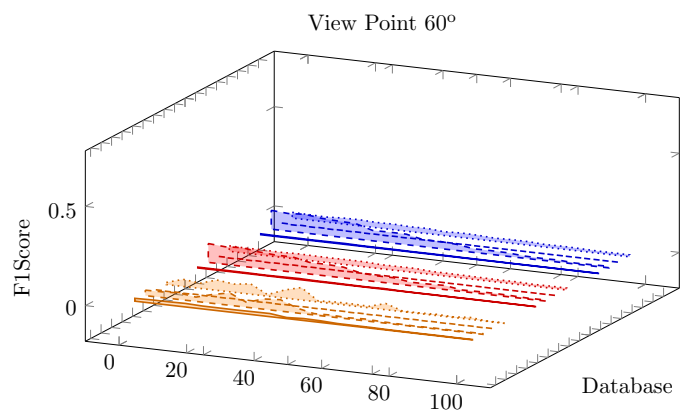

Percentage of pw-FPN

\begin{tabular}{l}
\hline Cross Check: — GRAF - - - BARK - - WALL …… BOAT \\
Brute Force: — GRAF - - B BARK - - WALL …… BOAT \\
FLANN: — GRAF - - BARK - - WALL …… BOAT
\end{tabular}

Figure 14 